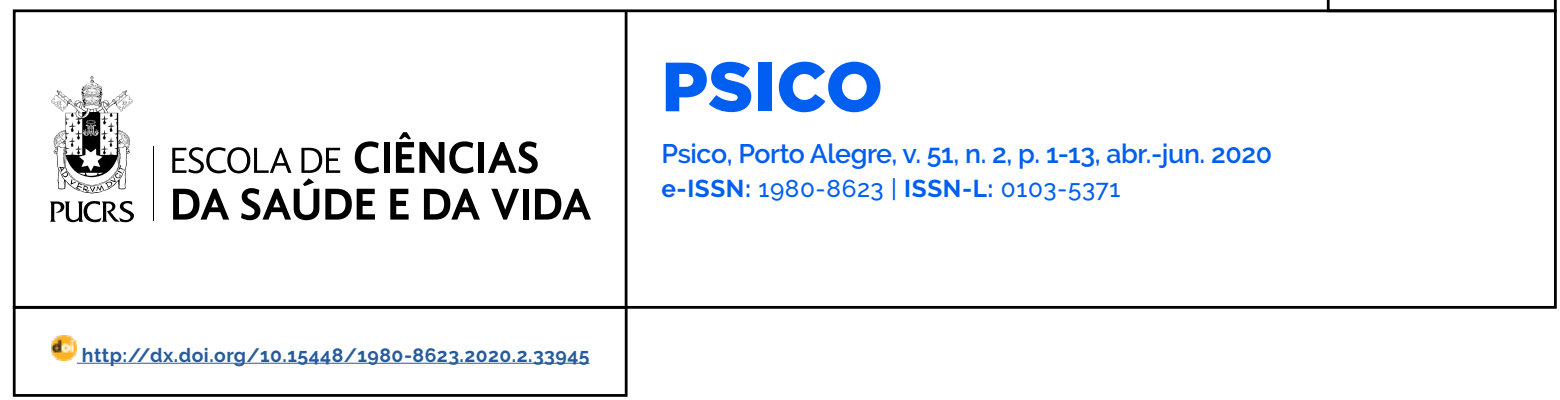

SEÇÃO: ARTIGO

\title{
O viés da aquiescência e a estrutura fatorial do Dirty Dozen e do Short Dark Triad
}

\author{
Acquiescence bias and the factor structure of the Dirty Dozen and the Short Dark Triad \\ El sesgo de la aquiescencia y la estructura factual del Dirty Dozen y del Short Dark Triad
}

\author{
Natália Costa Simões ${ }^{1}$ \\ orcid.org/0000-0002-2697-7851 \\ napsico17@hotmail.com
}

\author{
Nelson Hauck Filho ${ }^{1}$ \\ orcid.org/0000-0003-0121-7079 \\ hauck.nf@gmail.com
}

Recebido em: 17 abr. 2019

Aprovado em: 17 set. 2019

Publicado em: 4 set. 2020.
Artigo está licenciado sob forma de uma licença Creative Commons Atribuição 4.0 Internacional.
Resumo: A triade sombria - maquiavelismo, narcisismo e psicopatia - tem sido tradicionalmente avaliada via inventários de autorrelato. Entretanto, instrumentos de autorrelato podem estar suscetiveis à aquiescência ou tendência a concordar com itens mesmo quando possuem conteúdo antagônico. O presente estudo teve por objetivo investigar a dimensionalidade de dois populares instrumentos de autorrelato da triade. o Dirty Dozen e o Short Dark Triad, verificando a interferência do viés da aquiescência na estrutura fatorial de cada medida. Participaram do estudo 449 universitários (média de idades $=23,47 ; D P=6,76 ; 64 \%$ mulheres). O controle da aquiescência produziu soluções fatoriais mais interpretáveis do que a tradicional modelagem bi-factor, amplamente utilizada na área. Os achados também revelam fragilidades na composição teórica de ambos os instrumentos. Argumenta-se que a economia das medidas breves da "triade sombria" não deve vir desencontrada de uma estrutura fatorial que reflita o conhecimento atual sobre a multidimensionalidade desses traços.

Palavras-chave: avaliação psicológica, psicometria, triade sombria da personalidade.

Abstract: The Dark Triad - Machiavellianism, narcissism and psychopathy - has been traditionally assessed via self-report inventories. Self-report instruments might be susceptible to acquiescent responding or the tendency to manifest agreement with items even when they are semantically opposed. We investigated the dimensionality of two popular self-report instruments of the Dark Triad, the Dirty Dozen and the Short Dark Triad, and inspected the interference of the acquiescence bias in the factor structure of each measure. Participants were 449 students (mean age of 23.47 years; $S D=6.76 ; 64 \%$ females. Controlling for acquiescence produced factor solutions that were more theoretically interpretable than the standard bi-factor modeling, largely used in the field. Findings also raise questions about the theoretical underpinnings of both instruments. We argue that, despite valuable, brief measures of the Dark Triad traits should not come at the expense of a structure adequately reflecting the current knowledge about the multidimensionality of these traits.

Keywords: psychological assessment, psychometrics, dark triad.

Resumen: La Tríada Oscura (maquiavelismo, narcisismo y psicopatía) se ha evaluado a través de inventarios de autoinformes. El instrumento de autoinforme puede ser susceptible de responder o de la tendencia a manifestar acuerdo con los elementos, incluso cuando se oponen semánticamente.Investigamos la dimensionalidad de dos instrumentos populares de autoinforme de la Triada, Dirty Dozen y Short Dark Triad, e inspeccionamos la interferencia del sesgo de aquiescencia en la estructura de factores de cada medida. Los participantes fueron 449 estudiantes (edad promedio de 23.47 años; SD = 6.76; 64\% mujeres. El control de la aquiescencia produjo soluciones factoriales que eran teóricamente más fáciles de interpretar que el modelo bifactor estándar, ampliamente utilizado en el campo. Los hallazgos también plantean preguntas sobre los fundamentos teóricos de ambos instrumentos. Los rasgos de la Triada, no deben realizarse a expensas de una estructura que refleje adecuadamente el conocimiento actual sobre la multidimensionalidad de estos rasgos.

Palabras clave: evaluación psicológica, psicometria, triada oscura. 
Os traços de maquiavelismo, narcisismo e psicopatia compõem a chamada Tríade Sombria da Personalidade (Paulhus \& Williams, 2002). As variáveis da Tríade envolvem uma série de dificuldades interpessoais, tais como déficits de empatia, expressão e reconhecimento das emoções, manipulação interpessoal e dificuldade em dividir e cooperar com os outros (Jonason, Koenig, \& Tost, 2010; Jonason \& Middleton, 2015). Apesar de apresentarem um elo em comum, cada traço possui singularidades em relação aos demais. No maquiavelismo, destaca-se a orientação estratégica, a visão cínica do mundo e o pragmatismo; no narcisismo, a superioridade, a grandiosidade e a intitulação; na psicopatia, o desrespeito às regras sociais, a mentira patológica e a impulsividade (Jones \& Paulhus, 2014). Por estarem relacionados a problemas interpessoais fora do contexto clínico e forense, esses três traços têm sido considerados merecedores de investigação em amostras não clínicas (Furnham, Richards, \& Paulhus, 2013). Como o método do autorrelato é a estratégia mais usual em estudos na área (ex., Jakobwitz \& Egan, 2006; Jones \& Paulhus, 2017; Miller et al., 2012; Paulhus \& Jones, 2014; Vize, Collison, Miller, \& Lynam, 2018), vieses típicos do autorrelato podem ser uma preocupação na avaliação das características da tríade. Embora tenha havido certa preocupação com a influência da desejabilidade social (ver Ray et al., 2013), outras fontes de variância sistemática, como a aquiescência (isto é, concordar com o item em detrimento do conteúdo dele), têm sido pouco investigadas. O foco do presente estudo é na influência da aquiescência na estrutura fatorial de dois inventários que avaliam a Triade Sombria.

Os dois mais populares instrumentos de avaliação da Tríade Sombria da Personalidade são o Dirty Dozen (DD) (Jonason \& Webster, 2010) e o Short Dark Triad (SDT) (Jones \& Paulhus, 2014). O DD foi elaborado de forma a proporcionar uma avaliação rápida da Tríade Sombria, contendo apenas 12 itens pontuados em uma escala Likert de concordância ( 1 = discordo fortemente, 5 = concordo totalmente). Jonason e Webster (2010) relataram cargas fatoriais de 0,62 a 0,85 para as três escalas em uma série de estudos, além de estimativas de consistência interna de 0,63 a 0,78, o que pode ser considerado satisfatório para um instrumento com apenas quatro itens por fator. $O$ SDT, por sua vez, contém 27 itens e foi elaborado como uma alternativa ao DD, buscando oferecer uma melhor cobertura teórica aos componentes da triade (Jones \& Paulhus, 2014). Os autores buscaram incluir itens sobre estilo de vida errático e tendências antissociais à escala de psicopatia, dando assim maior cobertura teórica quando comparada à escala correspondente no DD, que somente inclui itens sobre insensibilidade. As estimativas de consistência interna das escalas do SDT encontradas pelos autores foram de 0,68 a 0,78 , o que pode ser considerado razoável.

Não obstante, a estrutura fatorial dos instrumentos DD e SDT tem sido objeto de intenso debate. Enquanto alguns estudos replicaram a estrutura original de três fatores do DD (Chiorri, Garofalo, \& Velotti, 2017; Gouveia, Monteiro, Gouveia, Athayde, \& Cavalcanti, 2016; Nascimento \& Gouveia, 2017), e do SDT (Atari \& Chegeni, 2016; Pechorro et al., 2018; Persson, Kajonius, \& Garcia, 2017), muitas investigações têm sugerido resultados diferentes. Por exemplo, o excesso de variância comum entre os itens de maquiavelismo e de narcisismo do SDT têm feito com que carreguem em um fator apenas (Persson et al., 2017; Rogoza \& Cieciuch, 2017). Além disso, de maneira geral, itens da tríade sombria tanto do DD quanto do SDT tendem a possuir uma grande parcela de variância comum. Por esse motivo, estudos mais recentes têm sido mais favoráveis a modelos de tipo bifator, que separam um fator geral - no qual todos os itens carregam e fatores específicos - nos quais apenas os itens de cada dimensão da triade carregam. Isso tem ocorrido tanto para o DD (Czarna, Jonason, Dufner, \& Kossowska, 2016; Kajonius, Persson, Rosenberg, \& Garcia, 2016; Maneiro, López-Romero, GómezFraguela, Cutrín, \& Romero, 2018; Savard, Simard, \& Jonason, 2017; Schimmenti et al., 2017), quanto para o SDT (Gamache, Savard, \& Maheux-Caron, 2018; McLarnon \& Tarraf, 2017; Shimotsukasa \& Oshio, 2017).

O fator geral da triade admite algumas interpretações teóricas. Evidências sugerem que 
a variância comum aos três traços representa a reduzida amabilidade (O'Boyle, Forsyth, Banks, Story, \& White, 2014), a tendência ao uso de táticas egocêntricas de relacionamento interpessoal e romântico (Jonason \& Kavanagh, 2010; Jonason, Li, \& Buss, 2010), a busca por dominância e poder (Jonason \& Ferrell, 2016), e a reduzida honestidade e humildade (Book et al., 2016). Sugere-se também que esses traços refletem, em parte, a expressão de um mesmo conjunto de genes (Vernon, Villani, Vickers, \& Harris, 2008). Vale ressaltar que, ao explorar a correlação latente entre o fator geral da tríade e o fator humildade-modéstia a partir de uma metanálise da literatura, Hodson et al. (2018) encontraram uma sobreposição quase perfeita entre essas variáveis $(r=-0,95)$. O elo entre a triade parece especialmente forte entre psicopatia e maquiavelismo, que apresentam também associações com falta de autocontrole (Jonason \& Tost, 2010) e com uma história de vida breve (Jonason et al., 2017).

Todavia, outra interpretação sobre um fator geral em dados sobre personalidade coletados via autorrelato também é admissivel. Como repetidamente demonstrado desde muitas décadas, estilos de resposta consistem em fontes de variância de erro sistemática que podem emergir enquanto um componente geral em modelos fatoriais (Bäckström \& Björklund, 2016; Bäckström, Björklund, \& Larsson, 2009; Edwards, 1969; Paulhus, 1981; Peabody, 1967; Wetzel \& Carstensen, 2015). Estilos de resposta podem ser entendidos como um fator de "método" (McCrae, 2018), e representam vieses no uso da escala de resposta de um instrumento, manifesto pela preferência por alguns pontos da escala em detrimento de outros (van Vaerenbergh \& Thomas, 2013). No contexto da avaliação de traços socialmente negativos, um dos principais focos de investigação tem sido a presença de respostas socialmente desejáveis, ou seja, aquelas com o objetivo de causar uma impressão favorável (Ray et al., 2013). Embora essa preocupação seja justificada em contextos de avaliação high-stakes, como uma entrevista de emprego, talvez outras fontes de variância erro sistemática mereçam mais atenção em situações de coleta anônima, quando inexistem incentivos para a adulteração de respostas.

Um dos estilos de resposta mais influentes, e que podem distorcer a estrutura fatorial de inventários de autorrelato é a aquiescência ou tendência a concordar com itens mesmo que tenham conteúdo antagônico (Paulhus, 1991). Respostas aquiescentes introduzem variância sistemática que pode confundir a estrutura fatorial de instrumentos de autorrelato (Kam \& Meyer, 2015; Schriesheim \& Eisenbach, 1995: Soto, John, Gosling, \& Potter, 2008). No caso de itens sobre psicopatologia, que são pouco endossados pela maioria das pessoas, essa tendência pode se manifestar enquanto desaquiescência, ou seja, mais discordar do que concordar, independentemente do nivel no traço apresentado pelo individuo. Como relatado por Kajonius et al. (2016) itens de autorrelato da tríade sombria podem apresentar distribuição bimodal, o que foi interpretado como fruto de repostas socialmente desejáveis. Todavia, a aquiescência/ desaquiescência também poderia produzir distribuições dessa natureza, motivo pelo qual deveria ser explorada de forma mais aprofundada.

Apesar da existência de muitas técnicas de controle estatístico da aquiescência (e.g., Kam \& Meyer, 2015; Maydeu-Olivares \& Coffman, 2006; Soto et al., 2008; Wetzel \& Carstensen, 2015), pouco se sabe a respeito do quanto esse viés impacta na estrutura fatorial de instrumentos avaliativos da triade. Além de a aquiescência potencialmente inflacionar a variância comum de um conjunto de itens positivamente correlacionados, ela pode diminuir a correlação entre itens positivos e negativos, mesmo quando avaliam um mesmo traço (Kam \& Meyer, 2015). Considerando que o instrumento DD contém apenas 12 itens positivos e o SDT contém 22 itens positivos e cinco itens negativos, a aquiescência/desaquiescência pode impactar de forma diferente a estrutura fatorial de cada um desses instrumentos. Mais especificamente, no DD, os itens podem resultar mais correlacionados entre si pela presença de variância de aquiescência, inflacionando suas cargas fatoriais. Enquanto isso, no SDT, as cargas 
dos itens negativos podem apresentar um padrão complexo e de dificil interpretação. Pode ocorrer de esses itens ficarem separados em um fator à parte ou, ainda, cargas de sinal contrário em dois fatores positivamente correlacionados. Em virtude disso, o objetivo do presente estudo foi investigara dimensionalidade de dois populares instrumentos de autorrelato da triade, o Dirty Dozen e o Short Dark Triad, verificando a interferência do viés da aquiescência na estrutura fatorial de cada medida. A hipótese levantada aqui é que uma parcela da variância comum aos itens desses instrumentos se deve à aquiescência/desaquiescência, e que controlar esse viés melhora a interpretabilidade da estrutura fatorial em cada caso.

\section{Método}

\section{Participantes}

Participaram desta pesquisa 449 estudantes universitários, sendo $64 \%$ mulheres, com média de idades 23,47 ( $D P=6,76$ anos). Os participantes eram provenientes de universidades de três estados brasileiros, sendo $36 \%$ do estado de Minas Gerais, 41\% do estado de São Paulo e 21,7\% do estado de Santa Catarina. A maioria declarouse branco $(66,2 \%)$ e solteiro $(51,3 \%)$.

\section{Instrumentos}

Dirty Dozen (DD) (Jonason \& Webster, 2010). O DD é um instrumento de autorrelato breve composto por 12 itens que avaliam traços de maquiavelismo ("Eu costumo manipular os outros para conseguir o que quero.", exemplo de item), psicopatia ("Eu costumo não sentir remorso", exemplo de item) e narcisismo ("Eu costumo querer que os outros prestem atenção em mim.", exemplo de item), sendo quatro itens para cada traço. Os itens são respondidos em uma escala Likert, 1 = Discordo totalmente a 5 = Concordo totalmente. No estudo original, a escala de psicopatia apresentou consistência interna (coeficiente $\alpha$ ) de 0,68, maquiavelismo 0,75, narcisismo 0,87.

\section{Short Dark Triad (SDT) (Jones \& Paulhus, 2014).}

O SDT é um inventário de autorrelato composto por 27 itens que avaliam traços de psicopatia ("Eu sei ser ruim para com os outros", exemplo de item), maquiavelismo ("É esperto manter informações que poderão ser usadas contra outras pessoas", exemplo de item) e narcisismo ("Muitas atividades em grupo tendem a ser chatas sem mim", exemplo de item), sendo nove itens para cada traço. Os itens são respondidos em uma escala Likert, 1 = Discordo totalmente a 5 = Concordo totalmente. No estudo original, a psicopatia apresentou consistência interna igual a 0,78, o maquiavelismo igual a 0,78, narcisismo 0,77, e a escala total 0,83.

\section{Procedimentos}

Inicialmente, foi feito contato com as universidades para apresentar o projeto de pesquisa e explicar os procedimentos de coleta, que foram realizados de maneira presencial. Após a aprovação das instituições por escrito, o projeto foi submetido para a avaliação do Comitê de Ética da Universidade São Francisco (CAAE: 53189716.7.0000.5514). Coma autorização do Comitê, as coletas foram agendadas de acordo com a disponibilidade dos professores, em sala de aula, coletivamente. Antes de iniciar as respostas aos instrumentos, os alunos assinaram um Termo de Consentimento Livre Esclarecido (TCLE) e, posteriormente, responderam ao questionário demográfico, ao DD e ao SDT. As aplicações duraram, em média, 20 minutos.

\section{Análises de dados}

Para cada instrumento, foram especificados três modelos candidatos, descritos na Figura 1 (para mais clareza de exposição, foram apresentados apenas dois itens por fator, e suprimidos os erros). No modelo 1, três fatores oblíquos (correlacionados entre si) explicam os itens dos instrumentos. No modelo 2, além dos três fatores especificos, um fator geral também explica os itens dos instrumentos. A parameterização é aquela tradicional de um modelo bifator, com todos os fatores ortogonais ao fator geral (e entre si). No modelo 3, os itens são explicados pelos três fatores obliquos e também por uma covariável, um indice de aquiescência, que busca dar conta da variância comum não atribuivel aos fatores de traço. Além disso, como também descrito na Figura 1, cada modelo foi testado seguindo duas 
diferentes especificações: restrito e saturado. Modelos restritos $(A)$ representam a tradicional abordagem confirmatória, em que cargas cruzadas são todas fixadas em zero, ou seja, proibidas de acontecer. Por sua vez, modelos saturados (B) são aqueles que permitem a existência de cargas cruzadas, o que não impede o item de carregar predominantemente em seu fator alvo. Como evidenciado em outros estudos, modelos de tipo A tendem a se mostrar representações implausiveis de dados sobre personalidade, de modo que modelos B, em geral, obtêm melhor ajuste (por exemplo, ver Aichholzer, 2014).

Figura 1 - Modelos candidatos testados

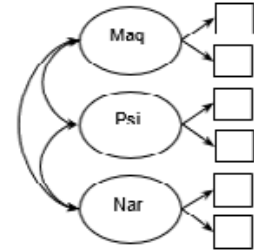

Trêz fatore obliquos

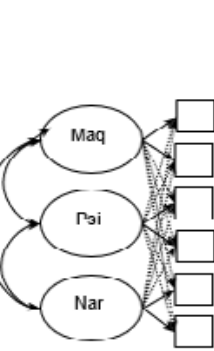

Três faturesubliquous

MODELOS A: especificaçāo restrita

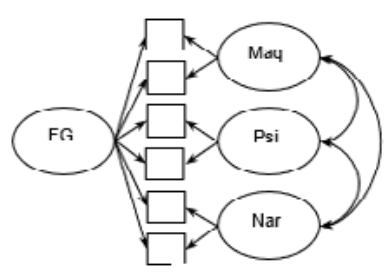

Bi fator

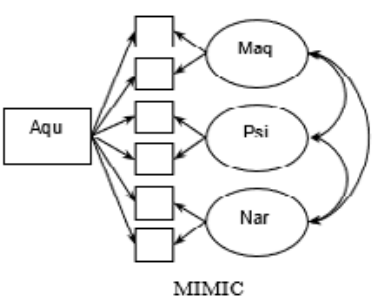

MODELOS B: especificação saturada

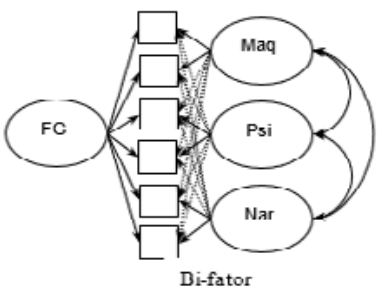

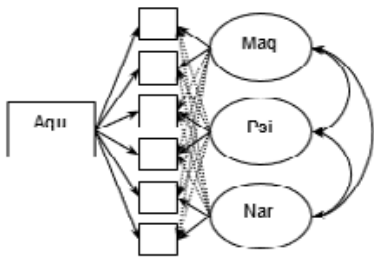

Mimic
Como o instrumento DD não possui itens semanticamente negativos (itens inversos), não foi possivel controlar a aquiescência com a estratégia dos modelos de intercepto randômico, como implementada e recomendada por Aicholzer (2014). Em vez disso, o controle aqui utilizado consistiu em uma adaptação da estratégia da ipsatização a partir de um indice de pares opostos (Hofstee, Berge, \& Hendriks, 1998). Primeiramente, escolhem-se pares opostos de itens para a composição do escore de aquiescência. A ideia é encontrar pares de itens com conteúdo oposto cuja média geral se aproxime do ponto central da escala (que é "3" para uma escala de cinco pontos). Do ponto de vista intraindivíduo, toda média desviante de "3" é indicativa de concordância com ou discordância do item em detrimento do conteúdo. Esse coeficiente então foi utilizado em um modelo de equações estruturais (MIMIC, ver Figura 1), servindo como variável de controle. $\mathrm{Na}$ composição do índice, foram levados em consideração oito itens do SDT: itens 11, 13, 14, 17 , 20, 22, 25 e 26. Esse conjunto de itens apresentou $M=3,00$ e $D P=0,43.0$ índice foi desenvolvido por meio dos itens apenas do SDT, pois o DD não apresenta itens positivos e negativos o que impossibilita a escolha e análise de pares opostos. Para o teste dos modelos, foi empregado o estimador Weighted Least Squares Mean and Variance adjusted. Para a avaliação do ajuste dos modelos, empregou-se o teste qui-quadrado e os índices de ajuste Root Mean Square Error of Approximation (valor de referência: RMSEA < 0,08), Confirmatory Fit Index (CFI > 0,95) e TuckerLewis Index (TLI > 0,95) (Hu \& Bentler, 1999)which includes using the maximum likelihood (ML. As análises foram conduzidas com os programas $\mathrm{R}$ e Mplus 7.11 (Muthén \& Muthén, 2014).

\section{Resultados}

A primeira etapa das análises foi o teste dos modelos candidatos. Os dados do DD produziram 
uma dificuldade: alguns itens apresentaram carga fatorial acima de 1,0, situação conhecida como "casos Heywood", indicando uma solução imprópria. Ao inspecionar os resultados preliminares mais cautelosamente, levantou-se a hipótese de que isso seria devido à presença de correlações residuais entre os itens quatro e cinco, sete e oito, e entre os itens nove e 10. Como esperado, essa modificação no modelo eliminou os casos Heywood, motivo pelo qual a especificação foi mantida em todos os modelos com o instrumento. Os indices de ajuste de cada modelo concorrente encontram-se dispostos na Tabela 1.

Tabela 1 - Ajuste dos modelos

\begin{tabular}{|c|c|c|c|c|c|c|}
\hline & $\chi^{2}$ & $g l$ & $p$ & CFI & TLI & RMSEA \\
\hline \multicolumn{7}{|l|}{ DD } \\
\hline Três fatores obliquos $\mathrm{A}$ & 251,91 & 48 & $<0,001$ & 0,970 & 0,959 & 0,097 \\
\hline Três fatores obliquos B & 93,95 & 31 & $<0,001$ & 0,991 & 0,980 & 0,067 \\
\hline Bi-factor A & 170,25 & 40 & $<0,001$ & 0,981 & 0,968 & 0,085 \\
\hline Bifactor B & 34,30 & 21 & $<0,034$ & 0,998 & 0,994 & 0,038 \\
\hline MIMIC A & 254,49 & 48 & $<0,001$ & 0,969 & 0,950 & 0,098 \\
\hline MIMIC B & 72,19 & 30 & $<0,001$ & 0,994 & 0,984 & 0,056 \\
\hline \multicolumn{7}{|l|}{ SDT } \\
\hline Três fatores obliquos $A$ & 1263,36 & 321 & $<0,001$ & 0,860 & 0,847 & 0,081 \\
\hline Três fatores obliquos B & 842,97 & 273 & $<0,001$ & 0,916 & 0,892 & 0,068 \\
\hline Bi-factor A & 1024,14 & 297 & $<0,001$ & 0,892 & 0,873 & 0,074 \\
\hline Bifactor B & 661,96 & 249 & $<0,001$ & 0,939 & 0,914 & 0,061 \\
\hline MIMIC A & 1169,63 & 321 & $<0,001$ & 0,877 & 0,856 & 0,077 \\
\hline MIMIC B & 788,92 & 273 & $<0,001$ & 0,926 & 0,897 & 0,065 \\
\hline
\end{tabular}

Como indicado em estudos prévios (ver Aichholzer, 2014), em todas as comparações, os modelos saturados ( $B$, aqueles que permitem cargas cruzadas) obtiveram melhor ajuste em relação aos seus respectivos concorrentes restritos. No caso do instrumento DD, não obstante, o ajuste dos três modelos saturados resultou bastante próximo, embora com uma pequena vantagem para o modelo bi-factor. Esse modelo produziu os menores valores qui-quadrado e RMSEA, e os maiores valores CFI e TLI. Quanto ao instrumento SDT, as diferenças em ajuste aos dados foram um pouco mais salientes, também favorecendo o modelo bi-factor, que produziu os menores valores qui-quadrado e RMSEA, e os maiores valores CFI e TLI. Todavia, ao serem inspecionadas as soluções produzidas pela modelagem bi-factor, em nenhum dos casos o padrão das cargas fatoriais foi consistente com a estrutura teórica (um fator geral e três fatores especificos independentes representando a tríade sombria). Por esse motivo, decidiu-se inspecionar melhores cargas fatoriais dos três modelos saturados de cada instrumento, descritas nas Tabelas 2 e 3. 
Tabela 2 - Soluções fatoriais produzidas para o DD

\begin{tabular}{|c|c|c|c|c|c|c|c|c|c|c|c|c|}
\hline \multirow{2}{*}{ Item } & \multirow{2}{*}{$\begin{array}{l}\text { Fator } \\
\text { teórico }\end{array}$} & \multicolumn{3}{|c|}{$\begin{array}{l}\text { Três fatores } \\
\text { obliquos }\end{array}$} & \multicolumn{4}{|c|}{ Modelo bifator } & \multicolumn{4}{|c|}{$\begin{array}{l}\text { MIMIC com controle de } \\
\text { aquiescência }\end{array}$} \\
\hline & & F1 & $\mathbf{F} 2$ & $\mathbf{F}_{3}$ & FG & F1 & $\mathbf{F 2}$ & F3 & Aqu & $\mathbf{F 1}$ & $\mathbf{F 2}$ & F3 \\
\hline $\begin{array}{l}\text { I1: manipular } \\
\text { os outros }\end{array}$ & M & 0,69 & $-0,06$ & 0,17 & 0,67 & 0,41 & $-0,07$ & 0,15 & 0,11 & 0,66 & $-0,01$ & 0,19 \\
\hline $\begin{array}{l}\text { I2: enganar e } \\
\text { mentir }\end{array}$ & M & 0,68 & 0,25 & $-0,02$ & 0,78 & 0,26 & 0,16 & $-0,02$ & 0,20 & 0,55 & 0,34 & 0,00 \\
\hline $\begin{array}{l}\text { I3: bajular as } \\
\text { pessoas }\end{array}$ & M & 0,62 & 0,02 & 0,21 & 0,71 & 0,15 & $-0,15$ & 0,16 & 0,17 & 0,53 & 0,10 & 0,23 \\
\hline $\begin{array}{l}\text { 14: aproveitar } \\
\text { dos outros }\end{array}$ & M & 0,51 & 0,42 & $-0,01$ & 0,83 & $-0,09$ & 0,14 & $-0,08$ & 0,14 & 0,36 & 0,57 & $-0,01$ \\
\hline $\begin{array}{l}\text { I5: não sentir } \\
\text { remorso }\end{array}$ & $P$ & 0,41 & 0,38 & 0,04 & 0,77 & $-0,19$ & 0,06 & $-0,06$ & 0,09 & 0,30 & 0,50 & 0,03 \\
\hline $\begin{array}{l}\text { I6: não impor- } \\
\text { tar com certo } \\
\text { ou errado }\end{array}$ & $P$ & 0,01 & 0,61 & 0,03 & 0,47 & $-0,19$ & 0,45 & $-0,02$ & 0,14 & $-0,22$ & 0,80 & 0,01 \\
\hline $\begin{array}{l}\text { I7: ser cruel e } \\
\text { insensivel }\end{array}$ & $P$ & $-0,01$ & 0,87 & $-0,02$ & 0,56 & 0,01 & 0,61 & $-0,05$ & 0,24 & $-0,00$ & 0,73 & $-0,06$ \\
\hline 18: ser cínico & $P$ & 0,25 & 0,54 & 0,01 & 0,62 & $-0,03$ & 0,05 & $-0,05$ & 0,18 & 0,30 & 0,41 & $-0,02$ \\
\hline $\begin{array}{l}\text { I9: querer } \\
\text { admiração }\end{array}$ & $\mathrm{N}$ & 0,03 & $-0,01$ & 0,75 & 0,44 & 0,04 & $-0,06$ & 0,59 & 0,13 & 0,05 & $-0,03$ & 0,73 \\
\hline $\begin{array}{l}\text { l10: querer que } \\
\text { prestem aten- } \\
\text { ção em mim }\end{array}$ & $\mathrm{N}$ & $-0,14$ & 0,00 & 0,87 & 0,36 & $-0,06$ & $-0,02$ & 0,69 & 0,06 & $-0,15$ & 0,01 & 0,86 \\
\hline $\begin{array}{l}\text { l11: buscar } \\
\text { prestígio }\end{array}$ & $\mathrm{N}$ & $-0,00$ & $-0,12$ & 0,85 & 0,35 & 0,15 & 0,01 & 0,77 & 0,18 & $-0,01$ & $-0,15$ & 0,86 \\
\hline $\begin{array}{l}\text { l12: esperar fa- } \\
\text { vores especiais }\end{array}$ & $\mathrm{N}$ & 0,12 & 0,11 & 0,59 & 0,54 & $-0,11$ & $-0,02$ & 0,47 & 0,13 & 0,04 & 0,17 & 0,59 \\
\hline \multicolumn{13}{|l|}{ Correlações } \\
\hline$F_{1}$ & & & 0,50 & 0.52 & 0 & & 0 & 0 & & & 0,51 & 0,48 \\
\hline $\mathrm{F} 2$ & & & & 0,32 & 0 & & & 0 & & & & 0,31 \\
\hline F3 & & & & & 0 & & & & & & & \\
\hline
\end{tabular}

Nota. $\mathrm{M}=$ Maquiavelismo, $\mathrm{P}=$ Psicopatia, $\mathrm{N}$ = Narcisismo. ${ }^{*}$ Caso Heywood (carga fatorial igual a ou acima de 1,0 ), indicando uma solução fatorial imprópria. 
Tabela 3 - Soluções fatoriais produzidas para o SDT

\begin{tabular}{|c|c|c|c|c|c|c|c|c|c|c|c|c|}
\hline \multirow{2}{*}{ Item } & \multirow{2}{*}{$\begin{array}{l}\text { Fator } \\
\text { teórico }\end{array}$} & \multicolumn{3}{|c|}{$\begin{array}{l}\text { Três fatores } \\
\text { obliquos }\end{array}$} & \multicolumn{4}{|c|}{ Modelo bifator } & \multicolumn{4}{|c|}{$\begin{array}{l}\text { MIMIC com controle de } \\
\text { aquiescência }\end{array}$} \\
\hline & & F1 & F2 & $F_{3}$ & FG & F1 & F2 & F3 & Aqu & $F_{1}$ & F2 & F3 \\
\hline $\begin{array}{l}\text { I1: não é esperto } \\
\text { contar segredos }\end{array}$ & $M$ & 0,34 & 0,13 & 0,24 & 0,35 & $-0,16$ & 0,18 & $-0,18$ & 0,20 & 0,39 & $-0,01$ & $-0,19$ \\
\hline $\begin{array}{l}\text { I2: manipular para } \\
\text { conseguir o que quer }\end{array}$ & $M$ & 0,52 & 0,02 & $-0,22$ & 0,62 & $-0,02$ & $-0,22$ & $-0,07$ & 0,06 & 0,41 & 0,30 & 0,15 \\
\hline $\begin{array}{l}\text { I3: pessoas importan- } \\
\text { tes a favor }\end{array}$ & $M$ & 0,50 & 0,03 & $-0,33$ & 0,62 & 0,09 & $-0,29$ & $-0,03$ & 0,10 & 0,33 & 0,34 & 0,28 \\
\hline $\begin{array}{l}\text { I4: evitar conflitos } \\
\text { com certas pessoas }\end{array}$ & $M$ & 0,44 & 0,37 & $-0,02$ & 0,58 & 0,20 & 0,03 & $-0,21$ & 0,27 & 0,49 & 0,06 & 0,21 \\
\hline $\begin{array}{l}\text { I5: manter informa- } \\
\text { ções para depois }\end{array}$ & $M$ & 0,71 & 0,07 & 0,00 & 0,75 & $-0,13$ & $-0,06$ & $-0,07$ & 0,17 & 0,52 & 0,35 & $-0,01$ \\
\hline $\begin{array}{l}\text { 16: hora certa de "dar } \\
\text { o troco" }\end{array}$ & $M$ & 0,77 & 0,01 & 0,10 & 0,76 & $-0,21$ & 0,03 & 0,01 & 0,19 & 0,47 & 0,42 & $-0,13$ \\
\hline $\begin{array}{l}\text { I7: esconder coisas } \\
\text { para manter reputação }\end{array}$ & $M$ & 0,50 & 0,33 & 0,22 & 0,57 & 0,01 & 0,21 & $-0,23$ & 0,33 & 0,54 & 0,02 & $-0,03$ \\
\hline $\begin{array}{l}\text { 18: certeza que os } \\
\text { planos o beneficiarão }\end{array}$ & $M$ & 0,62 & $-0,01$ & $-0,01$ & 0,60 & 0,00 & 0,00 & 0,16 & 0,21 & 0,18 & 0,47 & 0,06 \\
\hline $\begin{array}{l}\text { I9: maioria das pes- } \\
\text { soas manipuladas }\end{array}$ & $M$ & 0,34 & 0,18 & 0,03 & 0,41 & 0,06 & 0,04 & $-0,10$ & 0,18 & 0,31 & 0,10 & 0,07 \\
\hline 110: verdadeiro líder & $\mathrm{N}$ & 0,00 & 0,29 & $-0,42$ & 0,21 & 0,45 & $-0,27$ & $-0,12$ & 0,04 & 0,13 & $-0,01$ & 0,54 \\
\hline $\begin{array}{l}\text { I11: odeio o centro } \\
\text { das atenções }\end{array}$ & $\mathrm{N}$ & 0,18 & $-0,17$ & 0,34 & 0,01 & $-0,27$ & 0,26 & 0,18 & 0,28 & $-0,19$ & 0,15 & $-0,35$ \\
\hline $\begin{array}{l}\text { I12: atividades em } \\
\text { grupos chatas }\end{array}$ & $\mathrm{N}$ & 0,36 & 0,10 & $-0,14$ & 0,39 & 0,24 & $-0,05$ & 0,18 & 0,18 & $-0,01$ & 0,35 & 0,28 \\
\hline |13: pessoa especial & $\mathrm{N}$ & 0,02 & 0,52 & $-0,16$ & 0,18 & 0,72 & 0,08 & $-0,02$ & 0,39 & $-0,03$ & $-0,05$ & 0,62 \\
\hline $\begin{array}{l}\text { I4: apresentado a } \\
\text { pessoas importantes }\end{array}$ & $\mathrm{N}$ & 0,30 & 0,54 & $-0,13$ & 0,52 & 0,47 & 0,00 & $-0,20$ & 0,36 & 0,40 & $-0,02$ & 0,46 \\
\hline $\begin{array}{l}\text { I15: sem jeito com } \\
\text { elogio }\end{array}$ & $\mathrm{N}$ & 0,04 & 0,25 & 0,57 & $-0,07$ & $-0,03$ & 0,56 & 0,00 & 0.35 & $-0,03$ & $-0,15$ & $-0,27$ \\
\hline $\begin{array}{l}\text { I6: comparado a } \\
\text { pessoas famosas }\end{array}$ & $\mathrm{N}$ & 0,26 & 0,23 & $-0,22$ & 0,33 & 0,45 & $-0,07$ & 0,17 & 0,22 & $-0,08$ & 0,30 & 0,47 \\
\hline 117: pessoa comum & $\mathrm{N}$ & $-0,09$ & 0,01 & 0,50 & $-0,23$ & $-0,23$ & 0,41 & 0,00 & 0,23 & $-0,11$ & $-0,20$ & $-0,42$ \\
\hline $\begin{array}{l}\text { l18: respeito que } \\
\text { mereço }\end{array}$ & $N$ & 0,24 & 0,20 & 0,17 & 0,24 & 0,10 & 0,19 & 0,01 & 0,21 & 0,12 & 0,08 & 0,02 \\
\hline $\begin{array}{l}\text { I9: vingar de pesso- } \\
\text { as com autoridade }\end{array}$ & $P$ & 0,82 & $-0,26$ & $-0,01$ & 0,68 & $-0,09$ & $-0,01$ & 0,46 & 0,21 & $-0,06$ & 0,83 & 0,01 \\
\hline $\begin{array}{l}\text { I20: evito situações } \\
\text { perigosas }\end{array}$ & $P$ & $-0,03$ & 0,43 & 0,50 & $-0,03$ & 0,09 & 0,50 & $-0,20$ & 0,43 & 0,16 & $-0,36$ & $-0,16$ \\
\hline $\begin{array}{l}\text { I21: vingança rápida } \\
\text { e cruel }\end{array}$ & $P$ & 0,77 & $-0,23$ & 0,01 & 0,65 & $-0,13$ & 0,00 & 0,36 & 0,21 & 0,03 & 0,71 & $-0,04$ \\
\hline
\end{tabular}




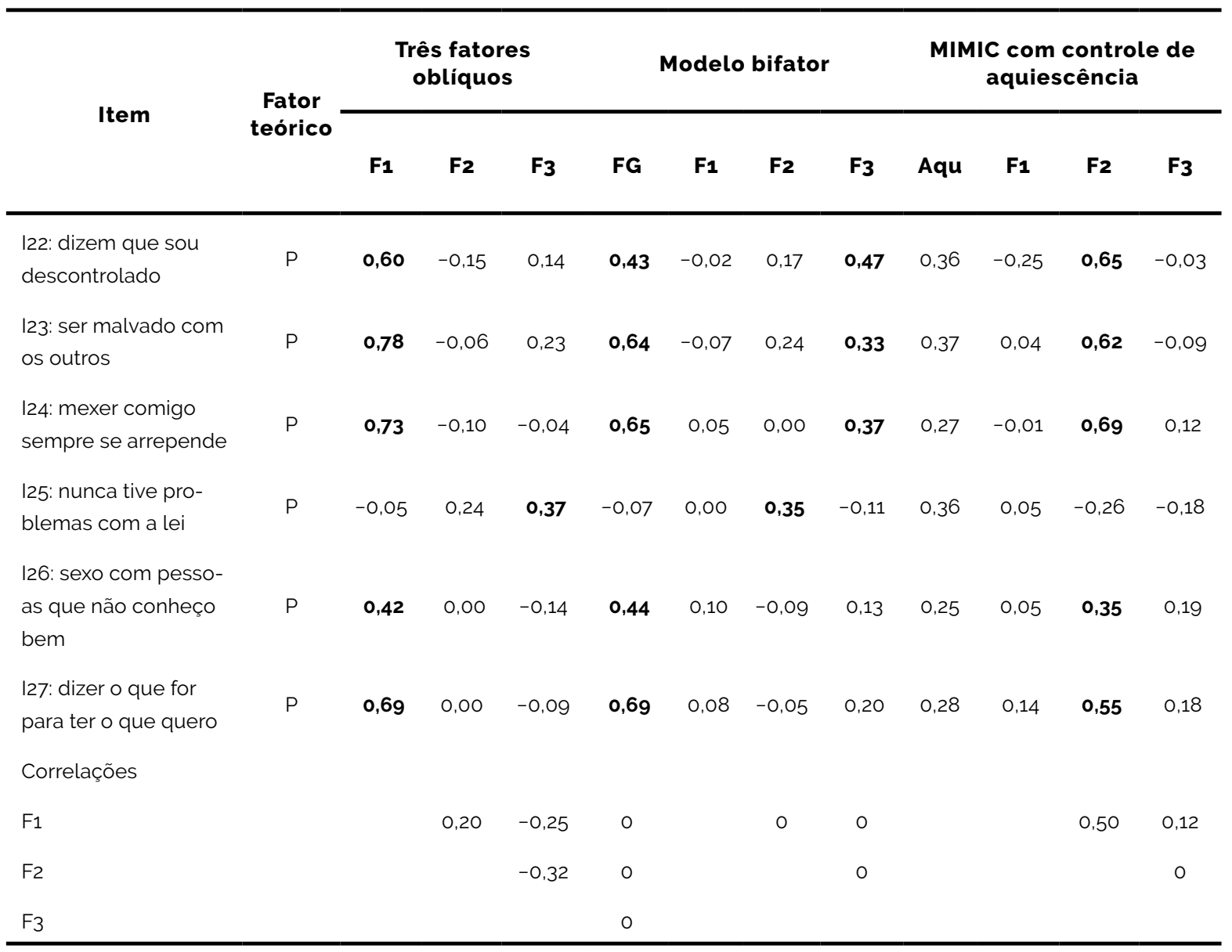

Nota. $\mathrm{M}$ = Maquiavelismo, $\mathrm{P}$ = Psicopatia, $\mathrm{N}$ = Narcisismo. No caso do DD, as soluções mais interpretáveis foram aquelas dos modelos de três fatores obliquos e três fatores obliquos com controle de aquiescência (MIMIC). A única diferença foi que, no modelo MIMIC, as cargas fatoriais - todas elas positivas, já que não há itens negativos - resultaram ligeiramente atenuadas em relação ao modelo sem controle de aquiescência. Em contraste, as estimativas paramétricas foram mais dificeis de interpretar no caso do SDT. O modelo de três fatores obliquos, por exemplo, parece ter consistido em um fator agrupando maquiavelismo e psicopatia, e outros dois fatores separando (embora não perfeitamente) entre itens de narcisismo positivos e reversos. Essa separação entre itens de acordo com a direção semântica ficou ainda mais evidente no modelo bi-factor. Nesse caso, o fator 2 é, claramente, aquele que reuniu os itens reversos, independentemente da dimensão teórica. Por sua vez, o modelo MIMIC levou alguma vantagem porque, apesar de algumas cargas cruzadas, os três fatores resultaram mais facilmente interpretáveis como a triade sombria. Nenhum fator de itens negativos foi produzido. Outro efeito do controle de aquiescência pode ser observado a partir das correlações entre os fatores dos modelos. No instrumento que contém itens negativos, o SDT, observa-se que houve uma alteração bastante expressiva nas correlações entre os fatores. A correlação entre maquiavelismo e psicopatia aumentou, e a correlação do narcisismo com os demais diminuiu, padrão associativo coerente com os achados de uma metanálise recente (Vize, Lynam, Collison, \& Miller, 2018).

\section{Discussão e considerações finais}

O presente estudo buscou aprofundar a discussão em torno da estrutura interna de dois famosos instrumentos de autorrelato que avaliam a Triade Sombria da Personalidade - o Dirty Dozen e o Short Dark Triad. A principal hipótese desse estudo foi que a presença de aquiescência contribuiria para a distorção da estrutura fatorial dessas escalas, o que foi confirmado pelas análises.
Além disso, conforme hipotetizado, observa-se que o efeito desse estilo de resposta se deu de maneira diferente em cada instrumento. No instrumento DD, controlar a aquiescência resultou em uma ligeira redução nas cargas dos quatro itens do fator maquiavelismo, de dois itens do fator psicopatia e de dois itens do fator narcisismo. Esse é exatamente o efeito esperado quando todos os itens de um instrumento são semanticamente 
positivos e, portanto, seus escores estão confundidos com a aquiescência (Schriesheim \& Eisenbach, 1995). Já no caso do SDT, que contém cinco itens reversos, o efeito foi bastante distinto. Enquanto ambos os modelos de três fatores obliquos e bi-factor com três fatores especíicos produziram fatores de método, o mesmo não foi observado no modelo MIMIC. A aquiescência, por atuar no sentido da concordância mesmo com itens semanticamente inversos, atenuou a correlação entre itens reversos e seus demais itens (positivos) do mesmo fator. O efeito disso pôde ser observado na separação desses itens em um fator à parte, agrupados à revelia de sua dimensão teórica original. Nos modelos obliquo e bi-factor, pelo menos um fator atraiu todos ou quase todos os itens semanticamente negativos. Isso não ocorreu no modelo MIMIC, o que ilustra como esse controle, de fato, melhora a interpretabilidade de instrumentos de autorrelato da Triade Sombria da Personalidade (Kam \& Meyer, 2015; Schriesheim \& Eisenbach, 1995; Soto et al., 2008).

Embora o controle efetuado tenha melhorado a interpretabilidade da estrutura, ele também levantou questionamentos sobre a cobertura teórica oferecida para cada dimensão da triade no DD e no SDT. Os dois inventários não parecem conter uma seleção de itens unidimensionais que apresentem variância comum referente a apenas um fator da triade sombria da personalidade. Confirmando resultados de outras investigações (Persson et al., 2017; Rogoza \& Cieciuch, 2017), itens de maquiavelismo e de psicopatia resultaram menos diferenciados em suas cargas do que o esperado. Embora o controle da aquiescência tenha tornado os fatores dos dois instrumentos mais interpretáveis, ainda assim permaneceram cargas cruzadas de itens de maquiavelismo e psicopatia, o que pode ser visto nas Tabelas 2 e 3. Não obstante, essa falta de diferenciação não surpreende quando se atenta ao conteúdo dos itens. Tanto o DD quanto o SDT incluem, em seu fator de maquiavelismo, itens sobre mentira e manipulação, características clássicas da psicopatia (Cleckley, 1976; Hare \& Neumann, 2008; Patrick, Fowles, \& Krueger, 2009). É bastante improvável, portanto, que tais itens carreguem zero em um fator de psicopatia. De fato, uma metanálise recente da literatura indicou que, de fato, maquiavelismo representa um domínio específico da psicopatia, e não um traço independente (Vize, Lynam, Collison, \& Miller, 2018).

Outra fragilidade conceitual merece atenção. Supostamente, o SDT apresenta a "vantagem" de que seu fator de psicopatia contém itens sobre impulsividade e descontrole, importantes caracteristicas não contempladas no DD (Jones \& Paulhus, 2014). Entretanto, esses itens encontram-se alocados em apenas um fator de psicopatia, junto aos demais itens sobre insensibilidade, o que faz pouco sentido. Tanto a pesquisa psicométrica quanto as neurociências sugerem que a insensibilidade/ manipulação e a impulsividade/antissocialidade da psicopatia constituem traços distintos, com uma base neurobiológica própria (ver Fowles \& Dindo, 2009). Naturalmente, pode não se sustentar, empiricamente, a junção desses fenômenos - por decreto - em uma mesma dimensão teórica. Se esses fenômenos possuem uma causa latente diversa, então eles aparecerão separados na análise fatorial. Dessa forma, a economia de ter medidas breves que avaliam a "Triade Sombria" parece vir ao custo de uma estrutura fatorial que possa refletir o conhecimento atual sobre a estrutura e a multidimensionalidade desses traços.

Confirmando estudos prévios, o modelo bifactor obteve um bom ajuste aos dados (Czarna et al., 2016; Gamache et al., 2018; Kajonius et al., 2016; Maneiro et al., 2018; McLarnon \& Tarraf, 2017; Savard et al., 2017; Schimmenti et al., 2017; Shimotsukasa \& Oshio, 2017). Todavia, um comentário adicional merece ser feito a respeito desse modelo. Como se pode observar nas Tabelas 2 e 3, o fator geral prevaleceu fortemente sobre os fatores especificos, sobrando pouca variância residual para esses em comparação àquele. Todavia, fatores gerais extraidos de inventários de personalidade tendem a concentrar variância valorativa, mais conhecida como "desejabilidade social" (Bäckström \& Björklund, 2016; Bäckström et al., 2009; Edwards, 1969; Peabody, 1967; Wetzel \& Carstensen, 2015). Por isso, a interpretação substantiva desse fator geral como sendo um elo comum entre a triade não deve ser lançada sem cautela. Além disso, 
uma metanálise mostrou que, ao residualizar a variância da triade controlando pelo fator geral, são produzidos fatores específicos de difícil interpretação e de rede nomológica implausivel (Vize, Collison, et al., 2018). Portanto, seria importante que pesquisas futuras investigassem se esse fator geral da triade se mantém mesmo quando controlada a desejabilidade social, algo que tem se mostrado improvável em outros domínios da personalidade (Bäckström \& Björklund, 2016; Bäckström et al., 2009).

Por fim, um aspecto geral sobre o controle da aquiescência merece destaque. Embora os itens negativos do SDT tenham resultado em possiveis fatores de método nos resultados aqui relatados, isso não significa que um inventário da triade não deva conter tais itens. Muito pelo contrário, é a disponibilidade de itens inversos que permite a implementação de técnicas como as descritas neste estudo. Vale ressaltar que o indice utilizado para corrigir a aquiescência em ambos os inventários foi composto unicamente por pares semanticamente opostos do SDT, dada a indisponibilidade desses pares no DD. A mensagem final é que as variâncias traço e aquiescência estarão fatalmente indissociadas no DD, exceto se a informação externa ao instrumento seja incluída no modelo para efetuar essa separação - como aqui foi feito. Exatamente em função dessa limitação do DD, não pôde ser utilizado o modelo de interceptos randômicos (A. Maydeu-Olivares \& Coffman, 2006), que teria sido uma estratégia mais refinada de análise da aquiescência. Para além de uma investigação centrada no efeito de um estilo de resposta, o presente estudo busca lançar luz em aspectos teóricos da Tríade Sombria. Argumenta-se aqui que o fortalecimento da teoria na área depende. fundamentalmente, da eliminação prévia de componentes de variância que podem distorcer a estrutura dos fenômenos.

\section{Referências}

Aichholzer, J. (2014). Random intercept EFA of personality scales. Journal of Research in Personality, 53, 1-4. https://doi.org/10.1016/j.jrp.2014.07.001
Atari, M., \& Chegeni, R. (2016). Assessment of dark personalities in Iran: Psychometric evaluation of the Farsi translation of the Short Dark Triad (SD3-F). Personality and Individual Differences, 102, 111-117. https://doi.org/10.1016/j.paid.2016.06.070

Bäckström, M., \& Björklund, F. (2016). Is the general factor of personality based on evaluative responding? Experimental manipulation of item-popularity in personality inventories. Personality and Individual Differences, 96. 31-35. https://doi.org/10.1016/j.paid.2016.02.058

Bäckström, M., Björklund, F., \& Larsson, M. R. (2009). Five-factor inventories have a major general factor related to social desirability which can be reduced by framing items neutrally. Journal of Research in Personality, 43(3), 335-344. https://doi.org/10.1016/j.jrp.2008.12.013

Book, A., Visser, B. A., Blais, J., Hosker-Field, A., Methot-Jones, T., Gauthier, N. Y., ... D'Agata, M. T. (2016). Unpacking more "evil": What is at the core of the dark tetrad? Personality and Individual Differences, 90, 269-272. https://doi.org/10.1016/j.paid.2015.11.009

Chiorri, C., Garofalo, C., \& Velotti, P. (2017). Does the Dark Triad Manifest Similarly in men and Women? Measurement Invariance of the Dirty Dozen across sex. Current Psychology, in press. https://doi.org/10.1007/s12144-017-9641-5

Cleckley, H. (1976). The mask of sanity (5th ed.). St. Louis, MO: Mosby.

Czarna, A. Z., Jonason, P. K., Dufner, M., \& Kossowska, M. (2016). The Dirty Dozen Scale: Validation of a Polish Version and Extension of the Nomological Net. Frontiers in Psychology, 7. https://doi.org/10.3389/fpsyg.2016.00445

Edwards, A. L. (1969). Trait and Evaluative Consistency in Self-Description. Educational and Psychological Measurement, 29(4), 737-752. https://doi. org/10.1177/001316446902900401

Fowles, D. C., \& Dindo, L. (2009). Temperament and Psychopathy: A Dual-Pathway Model. Current Directions in Psychological Science, 18(3), 179-183. https://doi. org/10.1111/j.1467-8721.2009.01632.x

Furnham, A., Richards, S. C., \& Paulhus, D. L. (2013). The Dark Triad of Personality: A 10 Year Review. Social and Personality Psychology Compass, 3(7), 199-216. https:// doi.org/10.1111/spc3.12018

Gamache, D., Savard, C., \& Maheux-Caron, V. (2018). French adaptation of the Short Dark Triad: Psychometric properties and a head-to-head comparison with the Dirty Dozen. Personality and Individual Differences, 122(September 2017), 164-170. https://doi.org/10.1016/j.paid.2017.10.027

Gouveia, V. V., Monteiro, R. P., Gouveia, R. S. V., Athayde, R. A. A., \& Cavalcanti, T. M. (2016). Avaliando o lado sombrio da personalidade: Evidências psicométricas do dark triad dirty dozen. Interamerican Journal of Psychology, 50(3), 420-432.

Hare, R. D., \& Neumann, C. S. (2008). Psychopathy as a clinical and empirical construct. Annual Review of Clinical Psychology, 4, 217-46. https://doi.org/10.1146/ annurev.clinpsy.3.022806.091452 
Hodson, G., Book, A., Visser, B. A., Volk, A. A., Ashton, M. C., \& Lee, K. (2018). Is the Dark Triad common factor distinct from low Honesty-Humility? Journal of Research in Personality, 73, 123-129. https://doi.org/10.1016/j.jp.2017.11.012

Hofstee, W. K. B., Berge, J. M. F. T., \& Hendriks, A. A. J. (1998). How to score questionnaires. Personality and Individual Differences, 25(5), 897-909. https://doi. org/10.1016/S0191-8869(98)00086-5

Hu, L., \& Bentler, P. M. (1999). Cutoff criteria for fit indexes in covariance structure analysis: Conventional criteria versus new alternatives. Structural Equation Modeling: A Multidisciplinary Journal, 6(1), 1-55. https:// doi.org/10.1080/10705519909540118

Jakobwitz, S., \& Egan, V. (2006). The dark triad and normal personality traits. Personality and Individual Differences, 40(2), 331-339. https://doi.org/10.1016/j.paid.2005.07.006

Jonason, P. K., \& Ferrell, J. D. (2016). Looking under the hood: The psychogenic motivational foundations of the Dark Triad. Personality and Individual Differences, 94, 324-331. https://doi.org/10.1016/..paid.2016.01.039

Jonason, P. K., Foster, J. D., Egorova, M. S., Parshikova, O., Csathó, A., Oshio, A., \& Gouveia, V. V. (2017). The Dark Triad Traits from a Life History Perspective in Six Countries. Frontiers in Psychology, 8. https://doi. org/10.3389/fpsyg.2017.01476

Jonason, P. K., \& Kavanagh, P. (2010). The dark side of love: Love styles and the Dark Triad. Personality and Individual Differences, 49(6), 606-610. https://doi. org/10.1016/j.paid.2010.05.030

Jonason, P. K., Koenig, B. L., \& Tost, J. (2010). Living a Fast Life: The Dark Triad and Life History Theory. Human Nature, 21(4), 428-442. https://doi.org/10.1007/ S12110-010-9102-4

Jonason, P. K., Li, N. P., \& Buss, D. M. (2010). The costs and benefits of the Dark Triad: Implications for mate poaching and mate retention tactics. Personality and Individual Differences, 48(4), 373-378. https://doi.org/10.1016/j.paid.2009.11.003

Jonason, P. K., \& Middleton, J. P. (2015). Dark Triad: The "Dark Side" of Human Personality. In J. D. Wright (Ed.), International Encyclopedia of the Social \& Behavioral Sciences (2nd ed., pp. 671-675). Elsevier. https://doi. org/10.1016/B978-0-08-097086-8.25051-4

Jonason, P. K., \& Tost, J. (2010). I just cannot control myself: The Dark Triad and self-control. Personality and Individual Differences, 49(6), 611-615. https://doi. org/10.1016/j.paid.2010.05.031

Jonason, P. K., \& Webster, G. D. (2010). The dirty dozen: A concise measure of the dark triad. Psychological Assessment, 22(2), 420-432. https://doi.org/10.1037/a0019265

Jones, D. N., \& Paulhus, D. L. (2014). Introducing the short Dark Triad (SD3): a brief measure of dark personality traits. Assessment, 21(1), 28-41. https://doi. org/10.1177/1073191113514105

Jones, D. N., \& Paulhus, D. L. (2017). Duplicity among the dark triad: Three faces of deceit. Journal of Personality and Social Psychology, 113(2), 329-342. https:// doi.org/10.1037/psppoo00139
Kajonius, P. J., Persson, B. N., Rosenberg, P., \& Garcia, D. (2016). The (mis)measurement of the Dark Triad Dirty Dozen: exploitation at the core of the scale. PeerJ, 4 , e1748. https://doi.org/10.7717/peerj.1748

Kam, C. C. S., \& Meyer, J. P. (2015). How Careless Responding and Acquiescence Response Bias Can Influence Construct Dimensionality. Organizational Research Methods, 18(3), 512-541. https://doi. org/10.1177/1094428115571894

Maneiro, L., López-Romero, L., Gómez-Fraguela, J. A., Cutrin, O., \& Romero, E. (2018). Pursuing the Dark Triad. Journal of Individual Differences, 1-9. https://doi. org/10.1027/1614-0001/a000274

Maydeu-Olivares, A., \& Coffman, D. L. (2006). Random intercept item factor analysis. Psychological Methods, 11(4), 344-62. https://doi.org/10.1037/1082-989X.11.4.344

Maydeu-Olivares, A., \& Coffman, D. L. (2006). Random intercept item factor analysis. Psychological Methods, 11(4), 344-362. https://doi.org/10.1037/1082-989X.11.4.344

McCrae, R. R. (2018). Method biases in single-source personality assessments. Psychological Assessment, 30(9), 1160-1173. https://doi.org/10.1037/pas0000566

McLarnon, M. J. W., \& Tarraf, R. C. (2017). The Dark Triad: Specific or general sources of variance? A bifactor exploratory structural equation modeling approach. Personality and Individual Differences, 112, 67-73. https:// doi.org/10.1016/j.paid.2017.02.049

Miller, J. D., Few, L. R., Seibert, L. A., Watts, A., Zeichner, A., \& Lynam, D. R. (2012). An examination of the Dirty Dozen measure of psychopathy: a cautionary tale about the costs of brief measures. Psychological Assessment, 24(4), 1048-53.https://doi.org/10.1037/a0028583

Muthén, L. K., \& Muthén, B. O. (2014). Mplus user's guide. (L. K. Muthén \& B. O. Muthén, Eds.). Los Angeles: Seven.

Nascimento, S., \& Gouveia, V. (2017). Dark Triad Dirty Dozen : Avaliando seus Parâmetros Via TRI. Psico-USF, 22(2), 299-308. https://doi.org/10.1590/141382712017220209

O'Boyle, E. H., Forsyth, D. R., Banks, G. C., Story, P. A., \& White, C. D. (2014). A Meta-Analytic Test of Redundancy and Relative Importance of the Dark Triad and Five-Factor Model of Personality. Journal of Personality, 83(6):644-64. https://doi.org/10.1111/jopy.12126

Patrick, C. J., Fowles, D. C., \& Krueger, R. F. (2009). Triarchic conceptualization of psychopathy: developmental origins of disinhibition, boldness, and meanness. Development and Psychopathology, 21(3), 913-938. https:// doi.org/10.1017/S0954579409000492

Paulhus, D. L. (1981). Control of social desirability in personality inventories: Principal-factor deletion. Journal of Research in Personality, 15(3), 383-388. https://doi. org/10.1016/0092-6566(81)90035-0

Paulhus, D. L. (1991). Measurement and control of response bias. In J. P. Robinson, P. R. Shaver, \& L. S. Wrighsman (Eds.), Measures of personality and social psychological attitudes (pp. 17-59). San Diego, CA: Academic Press. 
Paulhus, D. L., \& Jones, D. N. (2014). Measures of Dark Personalities. Measures of Personality and Social Psychological Constructs. Elsevier Inc. https://doi.org/10.1016/ B978-0-12-386915-9.00020-6

Paulhus, D. L., \& Williams, K. M. (2002). The dark triad of personality: Narcissism, machiavellianism and psychopathy. Journal of Research in Personality, 36, 556-563.

Peabody, D. (1967). Trait inferences: Evaluative and descriptive aspects. Journal of Personality and Social Psychology, 7(4. Pt.2), 1-18. https://doi.org/10.1037/ho025230

Pechorro, P., Caramelo, V., Oliveira, J. P., Nunes, C., Curtis, S. R., \& Jones, D. N. (2018). The Short Dark Triad (SD3): Adaptation and Psychometrics among At-Risk Male and Female Youths. Deviant Behavior, in press, 1-14. https:// doi.org/10.1080/01639625.2017.1421120

Persson, B. N., Kajonius, P. J., \& Garcia, D. (2017). Revisiting the Structure of the Short Dark Triad. Assessment, in press. https://doi.org/10.1177/1073191117701192

Ray, J. V. Hall, J., Rivera-Hudson, N., Poythress, N. G., Lilienfeld, S. O., \& Morano, M. (2013). The relation between self-reported psychopathic traits and distorted response styles: a meta-analytic review. Personality Disorders, 4(1), 1-14. https://doi.org/10.1037/a0026482

Rogoza, R., \& Cieciuch, J. (2017). Structural Investigation of the Short Dark Triad Questionnaire in Polish Population. Current Psychology, 38, 756-763. https://doi. org/10.1007/s12144-017-9653-1

Savard, C., Simard, C., \& Jonason, P. K. (2017). Psychometric properties of the French-Canadian version of the Dark Triad Dirty Dozen. Personality and Individual Differences, 119, 122-128. https://doi.org/10.1016/j.paid.2017.06.044

Schimmenti, A., Jonason, P. K., Passanisi, A., la Marca, L., Di Dio, N., \& Gervasi, A. M. (2017). Exploring the Dark Side of Personality: Emotional Awareness, Empathy, and the Dark Triad Traits in an Italian Sample. Current Psychology, 1-10. https://doi.org/10.1007/s12144-017-9588-6

Schriesheim, C. A., \& Eisenbach, R. J. (1995). An Exploratory and Confirmatory Factor-Analytic Investigation of Item Wording Effects on the Obtained Factor Structures of Survey Questionnaire Measures. Journal of Management, 21(6), 1177-1193. https://doi. org/10.1177/014920639502100609

Shimotsukasa, T., \& Oshio, A. (2017). Development and Validation of the Japanese Version of the Short Dark Triad(SD3-J). The Japanese Journal of Personality, 26(1), 12-22. https://doi.org/10.2132/personality.26.1.2

Soto, C. J., John, O. P., Gosling, S. D., \& Potter, J. (2008). The developmental psychometrics of big five self-reports: Acquiescence, factor structure, coherence, and differentiation from ages 10 to 20. Journal of Personality and Social Psychology, 94(4), 718-737. https://doi. org/10.1037/0022-3514.94.4.718

van Vaerenbergh, Y., \& Thomas, T. D. (2013). Response Styles in Survey Research: A Literature Review of Antecedents, Consequences, and Remedies. International Journal of Public Opinion Research, 25(2), 195-217. https://doi.org/10.1093/ijpor/eds021
Vernon, P. A., Villani, V. C., Vickers, L. C., \& Harris, J. A. (2008). A behavioral genetic investigation of the Dark Triad and the Big 5. Personality and Individual Differences, 44(2), 445-452. https://doi.org/10.1016/j.paid.2007.09.007

Vize, C. E., Collison, K. L., Miller, J. D., \& Lynam, D. R. (2018). Examining the Effects of Controlling for Shared Variance among the Dark Triad Using Meta-analytic Structural Equation Modelling. European Journal of Personality, 32(1), 46-61. https://doi.org/10.1002/per.2137

Vize, C. E., Lynam, D. R., Collison, K. L., \& Miller, J. D. (2018). Differences among dark triad components: A meta-analytic investigation. Personality Disorders: Theory, Research, and Treatment, 9(2), 101-111. https:// doi.org/10.1037/per0000222

Wetzel, E., \& Carstensen, C. H. (2015). Multidimensional modeling of traits and response styles. European Journal of Psychological Assessment. https://doi. org/10.1027/1015-5759/a000291

\section{Natália Costa Simões}

Doutoranda em Psicologia pela Universidade São Francisco (USF, Campinas, SP, Brasil), pesquisadora bolsista CAPES da Universidade São Francisco, Campinas, SP, Brasil.

\section{Nelson Hauck Filho}

Doutor em Psicologia pela Universidade Federal do Rio Grande do Sul (UFRGS, Porto Alegre, RS, Brasil), professor no Programa de Pós-Graduação Stricto Sensu em Psicologia da Universidade São Francisco, Campinas, SP, Brasil.

\section{Endereço para correspondência}

Natália Costa Simões

Universidade São Francisco, Universidade São Francisco - Campus de Campinas.

Rua Waldemar César da Silveira, 105, Prédio 1, Laboratório 2 de Pós-Graduação

Jardim Cura D'Ars 13045510

Campinas, SP - Brasil 